\title{
Dynamic parameter adaptation in Ant Colony Optimization using a fuzzy system for TSP problems
}

\author{
Frumen Olivas, Fevrier Valdez, Oscar Castillo \\ Tijuana Institute of Technology \\ Tijuana, Mexico \\ frumen@msn.com, \{fevrier, ocastillo\}@ tectijuana.mx
}

\begin{abstract}
Fuzzy logic has been a useful tool for modeling complex problems, with the use of fuzzy variables and fuzzy rules, and in this paper we use a fuzzy system for parameter adaptation in the Ant Colony Optimization (ACO metaheuristic). In this case we perform the dynamic adaptation of Alpha and Rho parameters; this is to control the abilities of ACO to perform a global and local search. Simulation results show the advantage of the use of a fuzzy system for parameter adaptation.
\end{abstract}

Keywords: Fuzzy system, Ant colony optimization, dynamic parameter adaptation, ACO.

\section{Introduction}

A dynamic parameter adaptation for ACO using a fuzzy system is presented in this paper, using the idea of changing the parameters over the time, by using some metrics about the performance of the ACO algorithm; this is based on previous knowledge about some of the parameters in ACO, in this case Alpha and Rho parameters. With the modification of these parameters we want to control the exploration and exploitation abilities of ACO.

The main contribution of this paper is the development of a new method for parameter adaptation in ACO over the iterations, through the use of a fuzzy system, in this case the fuzzy system model the behavior of ACO by controlling its abilities of global and local search, simply by changing important parameters of ACO, like Alpha and Rho.

Dorigo introduced the method of Ant Colony Optimization in 1992 [15], developed by using the idea of collective intelligence in ants swarms or colonies, and modeling the process in which the ants find food, and bring this food to the nest, through the use of a trail substance or pheromone in real ants. ACO uses a population of artificial ants and each one represent a solution of a problem, and uses Eq. 1 to calculate the probabilities to select the next node, Eq. 2 to evaporate the pheromone trail, Eq. 3 to update the pheromone trail, and Eq. 4 to define how much pheromone is deposited by the best ants.

$$
P_{i j}^{k}=\frac{\left[\tau_{i j}\right]^{\alpha}\left[\eta_{i j}\right]^{\beta}}{\sum_{l} \in N_{i}^{k}\left[\tau_{i l}\right]^{\alpha}\left[\eta_{i l}\right]^{\beta}}, \quad \text { if } j \in N_{i}^{k}
$$

Where in Eq. 1, $\tau_{i j}$ is the pheromone trail associated to the arc $(i, j), \alpha$ determines the influence of the pheromone trail, $\eta_{i j}$ is an heuristic value related to the distance between cities $i$ and $j, \beta$ determine the effects of the heuristic information, and $N$ is the neighborhood of the ant $k$ when it is city $i$, this is, the set of cities that the ant $k$ haven't visited yet.

$$
\tau_{i j} \leftarrow(1-\rho) \tau_{i j}, \quad \forall(i, j) \in L
$$

In Eq. 2, $\rho$ is the percentage of evaporation of the pheromone trail, and $L$ is a set of all arcs in the graph.

$$
\tau_{i j} \leftarrow \tau_{i j}+\sum_{k=1}^{n} \Delta \tau_{i j}^{k}, \quad \forall(i, j) \in L
$$

In Eq. 3, $\Delta$ is the amount of pheromone deposited in the $\operatorname{arc}(i, j)$ by the ant $k$, defined in Eq. 4 .

$$
\Delta \tau_{i j}^{k}=\left\{\begin{array}{l}
\frac{1}{C^{k}}, \text { if the arc }(\mathrm{i}, \mathrm{j}) \text { belongs to } \mathrm{T}^{\mathrm{k}} \\
0, \text { otherwise }
\end{array}\right.
$$

In Eq. $4, C$ is the length of the tour constructed by the ant $k$, and $T$ is the tour constructed by the ant $k$.

ACO has had many improvements since it was first introduced, like Elitist Ant System (EAS) and Rank-based Ant System $\left(\mathrm{AS}_{\text {Rank }}\right)$, but these modifications only affect the way in how each ant deposits the pheromone, by adding an extra variable to the equation of pheromone update, in this case, and based on several experiments performed to determine the best method of ACO, and we use $\mathrm{AS}_{\text {Rank }}$, to implement our parameter adaptation.

The parameters that we want to control are Alpha and Rho, because alpha affects the diversity of the ant colony or the level in which the ants are separated or together each other, depending on the level of alpha, in the other hand, 
the rho parameter affect the memory of the ants because with a higher level of rho helps the ants to "forget" bad solutions and with a lower level help to maintains the best solutions.

Zadeh introduced fuzzy sets theory and fuzzy logic [12] and [13], and help us to model problems in an easy way, with membership functions [14], we can represent fuzzy sets and fuzzy rules represent the knowledge of an expert on the problem. The idea of using a fuzzy system is to control the abilities of exploration and exploitation of ACO, by modeling the problem with membership functions and fuzzy rules.

The traveling salesman problem (TSP) is the perfect benchmark to test ACO, because in part ACO was developed to solve this problem, so we use this as a test problem. This problem consists of a salesman which wants to visit all the cities represented in a graph, but only once, and starting from any city and return to the same city, so the best tour has the minimal cost or minimal travel distance (depending on the type of problem). Basically the TSP is about find a Hamiltonian cycle with minimal cost.

In the literature there are improvements and applications of ACO, Dorigo et al. [16] applied the method to TSP problems in Ant colony system: a cooperative learning approach to the traveling salesman problem, also in Ant colonies for the traveling salesman problem [17], The ant colony optimization meta-heuristic [18], and The ant system: optimization by a colony of cooperating agents [19], in which Dorigo et al. proposed improvements to the original method.

The main references in which we based our work are Practical genetic algorithms second edition published by Haupt et al. [22], Fundamentals of computational swarm intelligence by Engelbrecht et al. [2], and Neuro-fuzzy and soft computing: a computational approach to learning and machine intelligence by Jang et al. [9]. These references served as inspiration and give to us some ideas on how develop the proposed approach. Also in Nedjah et al. [21], they made a comparison of ACO and other optimization techniques, and the results of ACO are poor, so we want that ACO can improve the quality of its results.

The use of a fuzzy system to adapt some PSO parameters can be found in [3], and in [4] with a type-2 fuzzy system. In these papers can be observed that the use of a fuzzy system or a type-2 fuzzy system can help to improve the quality of the results of PSO, so we want to use of the same methodology in order to improve the results in ACO.

Another improvements in ant colony optimization are: Neyoy et al. [8] proposed a fuzzy system to control the convergence of ACO, Babuska et al. [10] proposed an ACO algorithm that has a fuzzy partitioning of the state space of the system, Yan et al. [11] proposed an ACO with fuzzy pheromone laying mechanism, Einipour et al. [1] a fuzzy-ACO method for detect breast cancer, Elloumi et al. [25] proposed an hybridation of fuzzy PSO and fuzzy ACO applied to TSP problems, Khan et al. [24] proposed a fuzzy ant colony optimization for topology design of distributed local area networks.

The rest of the paper is organized as follows: Section 2 describe the methodology used for the parameter adaptation in ACO, in Section 3 we analyze the results of apply our proposed approach to TSP problems, in Section 4 our conclusion are presented, and finally in Section 5 are listed the references used in this paper.

\section{Methodology}

This paper is a continuation of others methods previously developed, for example in [6] we propose a fuzzy system to adapt the alpha parameter, and in [5] we propose a fuzzy system to adapt rho parameter, so in this paper we propose a fuzzy system to adapt alpha and rho parameters, taking into account that these parameters interact with each other indirectly, but both affect in the control of the abilities of ACO to search global and local.

In order to design the fuzzy system used to control the adaptation of parameters in ACO over the iterations, we need to define some metrics about the iterations, so we use a percentage of the iterations elapsed, Eq. 5 describes the metric about the iterations, and also we use a metric about the diversity of the ants in the search space and is defined in Eq. 6.

$$
\text { Iteration }=\frac{\text { Current Iteration }}{\text { Maximum of Iterations }}
$$

Where in Eq. 5, current iteration is the number of elapsed iterations, and maximum of iterations is the number iterations established for ACO to find the best solution.

$$
\text { Diversity }(S(t))=\frac{1}{n_{s}} \sum_{i=1}^{n_{s}} \sqrt{\sum_{j=1}^{n_{x}}\left(x_{i j}(t)-\bar{x}_{j}(t)\right)^{2}}
$$

In Eq. $6, S$ is the colony or the population of ACO; $t$ is the current iteration or time, $n_{s}$ is the size of the colony, $i$ is the number of the ant, $n_{x}$ is the total number of dimensions, $j$ is the number of the dimension, $x_{i j}$ is the $j$ dimension of the ant $i, \bar{x}_{j}$ is the $j$ dimension of the current best ant of the colony.

The input and output variables are illustrated in Fig. 1, 2, 3 and 4 .

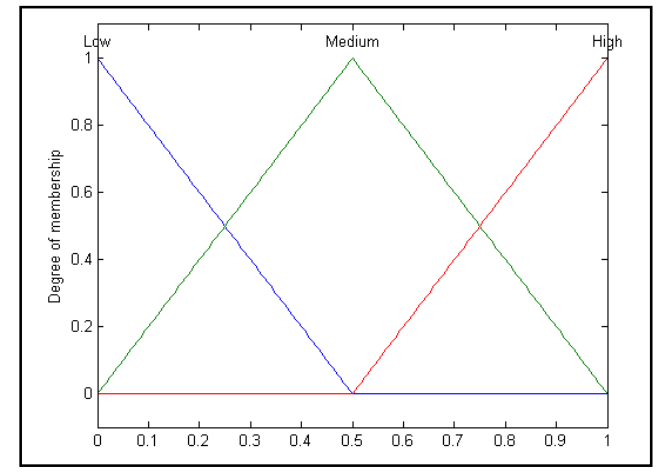

Fig. 1. Iteration as an input variable

Fig. 1 shows the iteration variable, with a range from 0 to 1 , and granulated into three triangular membership functions. 
The use of the percentage of the elapsed iterations helps us, in the model of the problem of parameter adaptation, because, with this input variable we have a manner to know if the method is just starting its iterations or is in its final iterations.

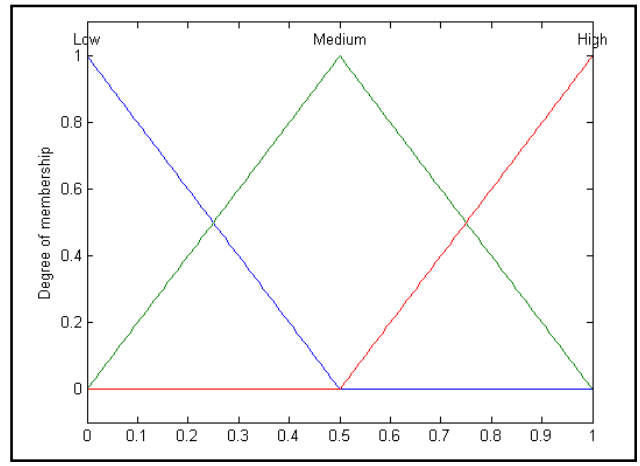

Fig. 2. Diversity as an input variable

In Fig. 2 the diversity variable is shown, with a range from 0 to 1 , and granulated into three triangular membership functions.

The granulation of the output variables is into five triangular membership functions, because we use the two input variables, which cause that the number of rules increase, and we need a more granulated output, to make a better model of the dynamic parameter adaptation.

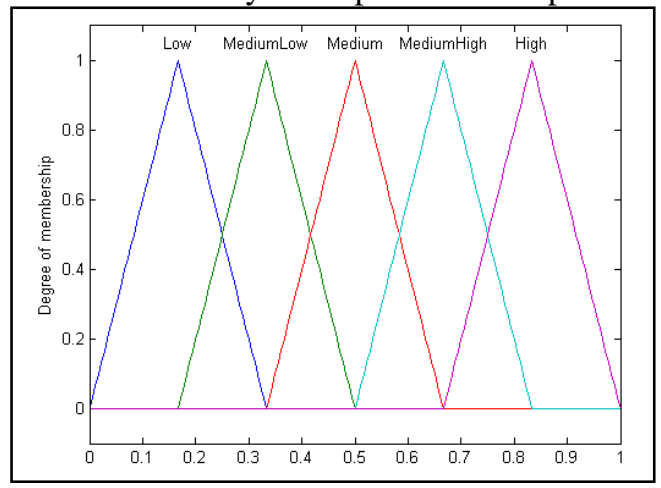

Fig. 3. Alpha parameter as output variable

Alpha as output variable is illustrated in Fig. 3, with a range from 0 to 1 , and granulated into five triangular membership functions. The range used for alpha is taken from [20], where values in the range of 0 to 1 are suggested.

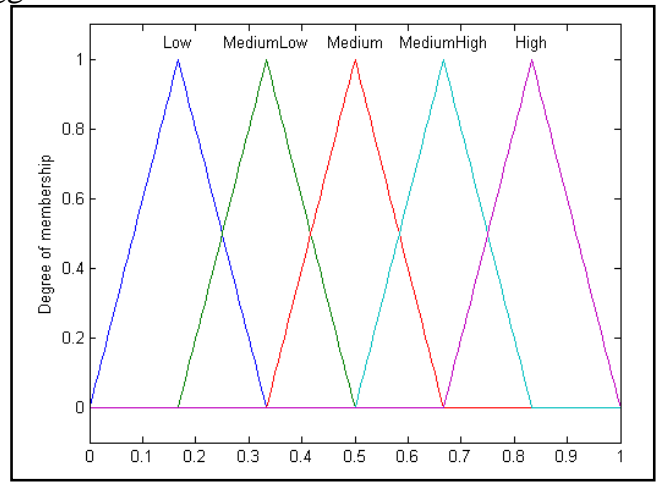

Fig. 4. Rho parameter as output variable
The output rho is shown in Fig. 4, with a range from 0 to 1 , and granulated into five triangular membership functions.

Once we have defined the possible inputs and outputs of the fuzzy system, we design a fuzzy system that includes all of these fuzzy variables, this is a fuzzy system with two inputs and two outputs illustrated in Fig. 5.

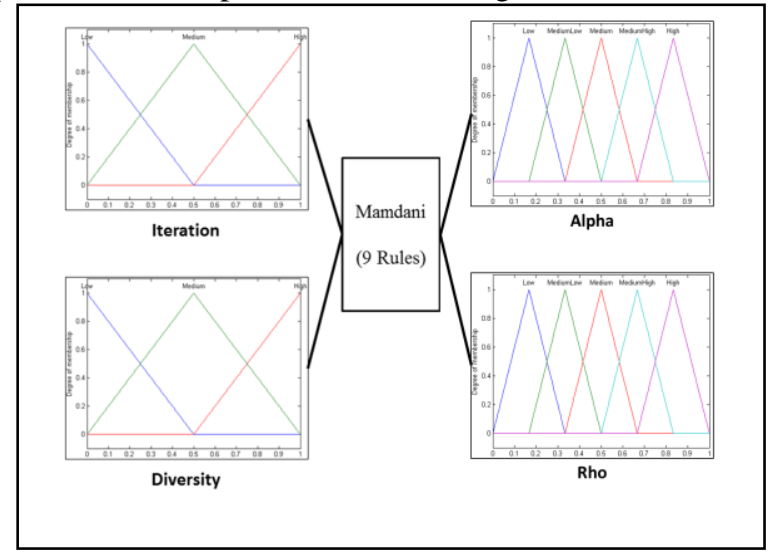

Fig. 5. Fuzzy system for parameter adaptation in ACO with iteration as input

The fuzzy system from Fig. 5, uses nine fuzzy rules that were developed using previous knowledge of the problem, this is, in early iterations the alpha parameter must be low, and rho must be high, so ACO can increase the exploration of the ants, and in final iterations alpha must be high and rho must be low, so the ants can exploit the best area found so far.

\begin{tabular}{|c|c|}
\hline 1. If (Iteration is Low) and (Diversity is Low) then & (Alpha is Low)(Rho is High) \\
\hline 2. If (Iteration is Low) and (Diversity is Medium) then & (Alpha is MediumLow)(Rho is MediumHigh \\
\hline 3. If (Iteration is Low) and (Diversity is High) then & (Alpha is Medium)(Rho is Medium) \\
\hline 4. If (Iteration is Medium) and (Diversity is Low) then & (Alpha is MediumLow)(Rho is MediumHigh \\
\hline 5. If (Iteration is Medium) and (Diversity is Medium) then & (Alpha is Medium)(Rho is Medium) \\
\hline 6. If (Iteration is Medium) and (Diversity is High) then & (Alpha is MediumHigh)(Rho is MediumLow \\
\hline 7. If (Iteration is High) and (Diversity is Low) then & (Alpha is Medium)(Rho is Medium) \\
\hline 8. If (Iteration is High) and (Diversity is Medium) then & (Alpha is MediumHigh)(Rho is MediumLow \\
\hline 9. If (Iteration is High) and (Diversity is High) then & (Alpha is High)(Rho is Low) \\
\hline
\end{tabular}

Fig. 6. Fuzzy rule set for the fuzzy system with iteration and diversity as inputs

The fuzzy rule set that is shown in Fig. 6, were developed based on previous knowledge about the parameters that we want to control, and in a combination of the previous systems, but basically is to control the abilities of search of ACO, now having two metrics about ACO that are percentage of the iterations and diversity of the colony. To give you an idea of how we develop this fuzzy rule set, here we have an example: if the percentage of iterations is low (this means on early iteration), and when the degree of dispersion of the particles is low (this means the colony of ants are closer to the best ant), then we need to increse the diversity or in other words use exploration, because we want to alow that the ants find another solutions, so to do that, we set alpha in low, and set rho in high so the ants can forget bad tours and increase the exploration of the space of search.

The pseudocode of the proposed approach is shown in Fig. 7, where the difference with respect to the ACO Rankbased is the agregation of line seven, because in this line 
we use a fuzzy system to calculate the new alpha and new rho parameters.

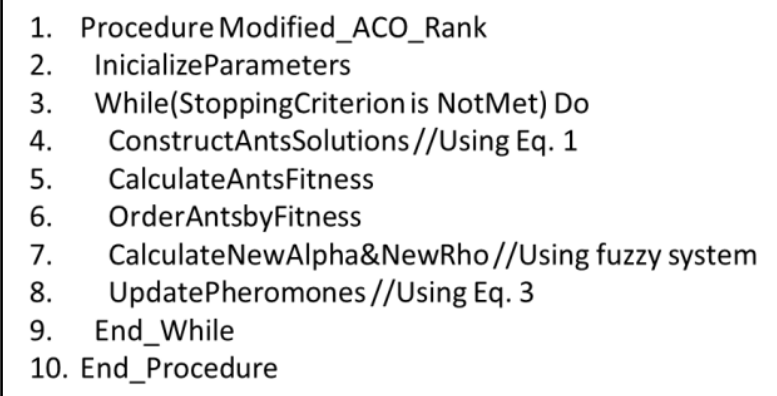

Fig. 7. Pseudocode of the modified ACO Rank-Based algorithm

With the inclusion of a fuzzy system to the ACO method it now involves more computational time, but this difference of time is not much when compared with the original method, but in order to improve the quality of the results, we are sacrifiying computational time for a better result. And we think that the proposed approach can achieve the same results as the original method in less iterations, but this statement has not been demonstrated yet, and maybe in future work we can demonstrate this statement.

Also we want to note that the individual modification of one parameter (with all other parameters fixed), is much more easier than trying to control two or more parameters at the same time, and this is because all the parameters have a direct or indirect interaction between them.

\section{Comparison of results}

To test the proposed approach, we use the TSP problem, the description of this problem is: given a set of nodes and distances for each pair of nodes, find a roundtrip of minimal total length visiting each node exactly once. And the distance from node $i$ to node $j$ is the same as from node $j$ to node $i$.

In order to perform the comparison, we use the same problems as in [5] and [6]. The problems used are taken from the TSPLIB [7], with the difference between them on the number of cities, in Table 1 are described the characteristics of the problems. Also after the table 1, the problems are described a little bit more.

\begin{tabular}{|l|c|c|}
\hline TSP & Number of Cities & Minimum \\
\hline Burma14 & 14 & 3323 \\
\hline Ulysses22 & 22 & 7013 \\
\hline Berlin52 & 52 & 7542 \\
\hline Eil76 & 76 & 538 \\
\hline KroA100 & 100 & 21282 \\
\hline
\end{tabular}

Table 1: Benchmark problems used to test the proposed method

Burma14 is a small TSP that consists of 14 pairs of data points from 14 cities of Burma, and the best known solution is a tour with the length of 3323 . Ulysses 22 is a TSP that consists of 22 locations from the Odyssey of Ulysses, and the best known solution is a tour with a length of 7013. Berlin52 is a TSP that contains 52 locations in Berlin, and the best known solution is a tour with a length of 7542 . Eil76 is a TSP that consists of 76 cities, and the best known solution is a tour with a length of 538 . KroA100 is a TSP that contains 100 cities, and the best known solution is a tour with a length of 21282 .

The methods to be compared are:

$\mathrm{AS}_{\mathrm{Rank}}$ : this is the original ACO Rank-based method.

$\mathrm{AS}_{\text {Rank }}+$ Alpha: this is the improved ACO Rank-based algorithm presented in [6].

$\mathrm{AS}_{\mathrm{Rank}}+\mathrm{Rho}$ : this is the improved ACO Rank-based algorithm presented in [5].

Proposed: this is the improved ACO Rank-based algorithm proposed in this paper.

The parameters used to test our approach in the TSP, are described in Table 2. And these parameters are the same as we use in [5] and [6].

\begin{tabular}{|l|c|c|c|c|}
\hline Parameter & $\mathbf{A S}_{\text {Rank }}$ & $\begin{array}{c}\mathbf{A S}_{\text {Rank }} \\
\text { +Alpha [6] }\end{array}$ & $\begin{array}{c}\mathbf{A S}_{\text {Rank }} \\
+ \text { Rho [5] }\end{array}$ & Proposed \\
\hline Alpha $\alpha$ & 1 & Dynamic & 1 & Dynamic \\
\hline Beta $\beta$ & 2 & 2 & 2 & 2 \\
\hline Rho $\rho$ & 0.1 & 0.1 & Dynamic & Dynamic \\
\hline Iterations & 1000 & 1000 & 1000 & 1000 \\
\hline Ants & $\begin{array}{c}\text { Num of } \\
\text { cities }\end{array}$ & Num of cities & $\begin{array}{c}\text { Num of } \\
\text { cities }\end{array}$ & $\begin{array}{c}\text { Num of } \\
\text { cities }\end{array}$ \\
\hline
\end{tabular}

Table 2: Parameters used to perform the experiments

The parameters from Table 2 were chosen based on several experiments that we performed in order to find the best parameters for $\mathrm{AS}_{\text {Rank }}$ without the parameter adaptation, and we use the same parameters for all methods in the comparison, and depending on the method, alpha or rho can be dynamic over the execution of ACO.

All the methods to be compared are applied to each TSP problem 30 times, and in Table 3 are the averages of these experiments for each TSP, performed with the parameters contained in Table 2.

\begin{tabular}{|l|c|c|c|c|}
\hline TSP & AS $_{\text {Rank }}$ & $\begin{array}{c}\text { AS }_{\text {Rank }} \\
\text { +Alpha [6] }\end{array}$ & $\begin{array}{c}\text { AS }_{\text {Rank }} \\
\text { +Rho [5] }\end{array}$ & Proposed \\
\hline Burma14 & 3350.3 & 3323 & 3323 & 3323 \\
\hline Ulysses22 & 7089.1 & 7013 & 7013 & 7013 \\
\hline Berlin52 & 7850.4 & 7549.3 & 7552.56 & 7542 \\
\hline Eil76 & 554.76 & 541.93 & 545.13 & 540.03 \\
\hline KroA100 & 22591 & 21470 & 21577.30 & 21404 \\
\hline
\end{tabular}

Table 3: Average results from the experiments with the TSPs

In Table 3 the results show that our proposed approach obtain on average better results when compared with all of the others methods. This is the proposed approach with a fuzzy system used to control alpha and rho parameters, compared with the original method with fixed parameters, and compared with the method that use a fuzzy system to control alpha, and also with the method with the fuzzy system to control rho.

There are more details from the results contained in Table 3; these details are the best result found by each method on each TSP, the worst result and the percentage of successful in each experiment, denoted by number of times in which the method found the global minimum over the maximum number of experiments. These details are shown in Table 4. 


\begin{tabular}{|c|c|c|c|c|}
\hline & $\mathbf{A S}_{\text {Rank }}$ & $\begin{array}{c}\mathbf{A S}_{\text {Rank }} \\
+ \text { Alpha [6] }\end{array}$ & $\begin{array}{c}\text { AS } \\
+ \text { Rho [5] }\end{array}$ & Proposed \\
\hline \multirow{5}{*}{ Best } & 3323 & 3323 & 3323 & 3323 \\
\hline & 7013 & 7013 & 7013 & 7013 \\
\hline & 7543 & 7542 & 7542 & 7542 \\
\hline & 539 & 538 & 538 & 538 \\
\hline & 22798 & 21294 & 21282 & 21282 \\
\hline \multirow{5}{*}{ Worst } & 3769 & 3323 & 3323 & 3323 \\
\hline & 7258 & 7013 & 7013 & 7013 \\
\hline & 8234 & 7760 & 7798 & 7542 \\
\hline & 586 & 598 & 560 & 549 \\
\hline & 23609 & 24381 & 21943 & 23942 \\
\hline \multirow{5}{*}{ Percentage } & $12 / 30$ & $30 / 30$ & $30 / 30$ & $30 / 30$ \\
\hline & $3 / 30$ & $30 / 30$ & $30 / 30$ & $30 / 30$ \\
\hline & $0 / 30$ & $29 / 30$ & $23 / 30$ & $30 / 30$ \\
\hline & $0 / 30$ & $8 / 30$ & $3 / 30$ & $15 / 30$ \\
\hline & $0 / 30$ & $0 / 30$ & $1 / 30$ & $5 / 30$ \\
\hline
\end{tabular}

Table 4: Details from the results of table 3

Table 4 shows that the proposed approach and the method $\mathrm{AS}_{\text {Rank }}+$ Rho can obtain at least one time the global minimum, but the proposed approach obtains a bigger successful rate in the last three TSP problems when compared with all of the others methods, and when compared only with the original method there are a bigger difference on successful rate, this is, the proposed approach can achieve more times the best known solution than all of the others methods.

Using as a reference the work in [23], we can perform a comparison using the z-test, between the proposed approach and the original ACO Rank-based, and against the other two methods that only controls alpha [6] or rho [5], in Table 5 the standard deviations from the 30 experiments for each TSP are shown, which average results are presented in Table 3 .

\begin{tabular}{|l|c|c|c|c|}
\hline TSP & AS $_{\text {Rank }}$ & $\begin{array}{c}\text { AS } \\
\text { +Alpha [6] }\end{array}$ & $\begin{array}{c}\text { AS }_{\text {Rank }} \\
+ \text { Rho [5] }\end{array}$ & Proposed \\
\hline Burma14 & 80.43 & 0 & 0 & 0 \\
\hline Ulysses22 & 50.09 & 0 & 0 & 0 \\
\hline Berlin52 & 179.25 & 39.80 & 46.84 & 0 \\
\hline Ei176 & 8.07 & 4.31 & 6.67 & 3.36 \\
\hline KroA100 & 486.67 & 118.33 & 175.20 & 37.68 \\
\hline
\end{tabular}

Table 5: Standard deviations from the results in table 3

From the results in Table 5, it can be observed that the proposed approach has the lowest standard deviations in all the TSP problems when compared with all of the other three methods. This means that our proposed approach obtains the lowers average results and even the lowers standard deviations in all of the TSP problems.

The results of the Z-test are presented in Table 6, where we made a comparison with the proposed approach against the other three methods, so the hypotheses are:

$H_{0}: \mu_{1} \geq \mu_{2}$

$H_{1}: \mu_{1}<\mu_{2}$

Where $\mu_{1}$ is the average of the proposed approach, $\mu_{2}$ is the average of the other methods. The null hypothesis $\left(H_{0}\right)$ means that the average of the proposed approach is bigger than the average of the other method; the alternative hypothesis $\left(H_{l}\right)$ means that the average of the proposed approach is lower than the average of the other method.
With a level of significance of $\alpha=0.05$, and a critical value of $Z_{0}=-1.96$, this means, the rejection region is for all values of $z$-test lower than $Z_{0}$.

The result NaN (Not a Number) in Table 6 means that a comparison isn't possible because the standard deviations of the two samples are zeros. Results in red are not in the rejection region, so there is not enough evidence at the 5\% level of significance to reject the null hypothesis. All other results are in the rejection region, so there is enough evidence at the $5 \%$ level of significance to support the alternative hypothesis and to reject the null hypothesis.

\begin{tabular}{|l|c|c|c|}
\hline TSP & $\mathbf{A S}_{\text {Rank }}$ & $\begin{array}{c}\text { AS } \\
\text { +Aank }\end{array}$ & $\begin{array}{c}\mathbf{A S}_{\text {Rank }} \\
+ \text { +Rho [5] }\end{array}$ \\
\hline Burma14 & -1.861 & $\mathrm{NaN}$ & $\mathrm{NaN}$ \\
\hline Ulysses22 & -8.321 & $\mathrm{NaN}$ & $\mathrm{NaN}$ \\
\hline Berlin52 & -9.424 & -0.999 & -1.235 \\
\hline Ei176 & -9.222 & -2.081 & -3.736 \\
\hline KroA100 & -14.103 & -6.017 & -7.441 \\
\hline
\end{tabular}

Table 6: Results of Z-test

Once we performed the statistical test, the results are presented in Table 6, where the proposed approach is compared with the other three methods, including the original ACO Rank-based algorithm, and the results show that only in the first TSP, there is no statistical evidence to say that our proposed approach obtains on average better results when compared with all other methods, however in the last two TSP problems, there is enough statistical evidence to state that our proposed obtains on average better results, this is, on TSP with more or equal than 76 cities, the proposed is better on finding a global minimum than the original method and the other methods with parameter adaptation.

\section{Conclusions}

The results obtained by the application of the four methods to the TSP problems are clear and we can state that the proposed method, obtains on average better results in the most complex problems, and we can conclude that the proposed method for dynamic parameter adaptation in Ant colony optimization rank-based or $\mathrm{AS}_{\text {Rank }}$ could improve the quality of the results when compared with the original algorithm, this is, our proposal obtains on average better results when compared with the other proposed method and also with the original algorithm.

Also from the results contained in Table 6, we can state, with a statistical basis, that the average of the proposed approach is lower than the other methods in the most complex TSPs.

For future work we want to level up the fuzzy system used in this paper, with the use of type-2 fuzzy logic to adapt the parameters of ant colony optimization, using the same methodology or even with another methodology, like a fuzzy controller.

\section{References}

[1] A. Einipour (2011). A fuzzy-ACO method for detect breast cancer Global Journal of Health Science, 3(2), p195.

[2] A. Engelbrecht (2005) "Fundamentals of Computational Swarm Intelligence", University of Pretoria, South Africa. 
[3] F. Olivas and O. Castillo (2012) "Optimal design of fuzzy classification systems using PSO with dynamic parameter adaptation through fuzzy logic", Elsevier, Expert systems with applications, 3196-3206.

[4] F. Olivas and O. Castillo (2013) "Particle swarm optimization with dynamic parameter adaptation using interval type-2 fuzzy logic for benchmark mathematical functions", Nature and Biologically Inspired Computing (NaBIC), 2013 World Congress on, 36-40.

[5] F. Olivas, F. Valdez and O. Castillo (2014) "Ant Colony Optimization with parameter adaptation using fuzzy logic for TSP problems", International Seminar on Computational Intelligence 2014, (Acepted).

[6] F. Olivas, F. Valdez and O. Castillo (2014) "Fuzzy system for parameter adaptation in Ant Colony Optimization", Swarm Intelligence (SIS), 2014 IEEE Symposium on, pp. 84-89.

[7] G. Reinelt (1991). TSP_LIB- A traveling Salesman Problem Library, ORSA Journal on Computing, 3, 376-384.

[8] H. Neyoy, O. Castillo and J. Soria (2012) "Dynamic fuzzy logic parameter tuning for ACO and its application in TSP Problems", SCI 451, pp. 259-271.

[9] J. Jang, C. Sun and E. Mizutani (1997), "Neuro-fuzzy and soft computing: a computational approach to learning and machine intelligence", Prentice-Hall, Upper Saddle River, NJ.

[10] J. Van Ast, R. Babuska and B. De Schutter "Fuzzy ant colony optimization for optimal control," Proceedings of the 2009 American Control Conference, St. Louis, Missouri, pp. 1003-1008, June 2009.

[11] L. Yu, J. Yan, G. Yan, and L. Yi (2012). ACO with Fuzzy Pheromone Laying Mechanism. In Emerging Intelligent Computing Technology and Applications (pp. 109-117). Springer Berlin Heidelberg.

[12] L. Zadeh (1965) "Fuzzy sets". Information \& Control, 8, 338-353.

[13] L. Zadeh (1988) "Fuzzy logic". IEEE Computer Mag., vol. 1, pp. 83-93.
[14] L. Zadeh (1975) "The concept of a linguistic variable and its application to approximate reasoning-I," Inform. Sci., vol. 8, pp. 199-249.

[15] M. Dorigo (1992) Optimization, Learning and Natural Algorithms, PhD Thesis, Dipartimento di Elettronica, Politechico di Milano, Italy.

[16] M. Dorigo and L. Gambardella (1997) "Ant Colony System: A Cooperative Learning Approach to the Traveling Salesman Problem", IEEE Transactions on Evolutionary Computing, 1, pp. 53-66.

[17] M. Dorigo and L. Gambardella (1997) "Ant Colonies for the Traveling Salesman Problem”, Biosystems, 43, pp. 73-81.

[18] M. Dorigo and G. Di Caro (1999) "The Ant Colony Optimization Meta-heuristic", in New Ideas in Optimization, Corne, D., Dorigo, M. and Golver, F. (eds), McGraw-Hill, pp. 11-32.

[19] M. Dorigo, V. Maniezzo and A. Colorni (1996) "The ant system: Optimization by a colony of cooperating agents" IEEE Trans. Syst, Man, Cybern. B, vol. 26, no. 2, pp. 29-41.

[20] M. Dorigo and T. Stutzle (2004) Ant Colony Optimization. MIT Press, Cambridge, MA.

[21] N. Nedjah (2006) "Swarm intelligent systems" (Vol. 26). Heidelberg: Springer.

[22] R. Haupt and S. Haupt (2004) "Practical Genetic Algorithms second edition", A Wiley-Interscience publication.

[23] R. Larson and B. Farber (2012). Elementary statistics: Picturing world (5th ed.). Boston, MA: Prentice Hall.

[24] S. Khan and A. Engelbrecht (2008). A fuzzy ant colony optimization algorithm for topology design of distributed local area networks. InSwarm Intelligence Symposium, 2008. SIS 2008. IEEE (pp. 1-7). IEEE.

[25] W. Elloumi, N. Baklouti, A. Abraham and A. Alimi (2013) "Hybridization of fuzzy PSO and fuzzy ACO applied to TSP", In Hybrid Intelligent Systems (HIS), 2013 13th International Conference on (pp. 105-110). IEEE. 